\title{
EFFECT OF THERMO-MECHANICAL CYCLIC LOADING ON THE FRACTURE RESISTANCE OF IMPLANT-SUPPORTED PROVISIONAL FDP FABRICATED BY CAD/CAM AND 3D PRINTING: AN IN-VITRO STUDY
}

\author{
Ahmed E. Bayoumi ${ }^{1 *}$, Hussein R Mohammed ${ }^{2}$, Tamer E. Shokry ${ }^{3}$
}

\begin{abstract}
Objective: This study evaluated the effect of thermo-mechanical cyclic loading (TMCL) on the fracture resistance of ImplantSupported Provisional Fixed Dental Prosthesis (ISP-FDP) fabricated by CAD/CAM and 3D printing. Material and Methods: Thirty ISP-FDPs were fabricated using 3D printing C\&B MFH (Crown \& Bridge Micro-filed Hybrid) and CAD/CAM. The samples were divided into two main groups of 15 samples according to fabrication technique. Each main group was divided into three equal sub-groupsaccording to the duration of thermo-mechanical cyclic loading (TMCL). The fracture resistance was then measured for all groups. Results: Two-way Analysis of Variance (ANOVA) showed that the fabrication technique (regardless of TMCL) had a statistically significant effect on mean fracture resistance (P-value $<0.001$, Effect size $=0.479)$. Thermo-mechanical cyclic loading (regardless of fabrication technique) had a statistically significant effect on mean fracture resistance (P-value $<0.001$, Effect size $=$ $0.743)$. The interaction between the two variables had a statistically significant effect on mean fracture resistance $(\mathrm{P}-\mathrm{value}=0.014$, Effect size $=0.299$ ). Since the interaction between the variables is statistically significant, so the variables are dependent upon each other. Conclusion: Fabrication technique impacted the fracture resistance of ISP-FDP. Thermo-mechanical cyclic loading caused degradation of mechanical properties in both materials. Both fabrication techniques offer a provisional prosthesis that can withstand normal functional loads for extended healing durations.
\end{abstract}

KEYWORDS: CAD/CAM, 3D printing, fracture resistance, temporary FDP, interim prosthesis.

\section{INTRODUCTION}

Provisional prosthesis is an essential component of fixed prosthodontic treatment. Whether to cover freshly cut dentin in conventional crown and bridgework or in advanced treatments like re-adjustment of the patient occlusion and implant cases $^{(1,2)}$. It should perform the same functions performed by definitive prosthesis only for a limited period of time.
Thus, provisional prostheses should attain ideal biological, mechanical, and esthetic properties. In addition, it should have good marginal integrity and polishability to allow guided tissue healing in implant cases $^{(3,4)}$.

Systematic reviews of implant success rates indicated that $74 \%$ of total failures occur in the first 12 months. The failure rate drops dramatically

1. Masters Candidate, Egyptian Ministry of Health.

2. Assistant Professor of Crown and Bridge, Faculty of Dental Medicine for Boys, Al-Azhar University.

3. Professor of Crown and Bridge, Faculty of Dental Medicine for Boys, Al-Azhar University.

-Corresponding author: ahmadsbayoumi@gmail.com

DOI: 10.21608 /ajdsm.2021.82890.1215 
after the first year ${ }^{(5)}$. This indicates long-term provisionalization of implant cases to weigh the success of proposed treatments. The oral environment poses great challenges on dental restorations by fluctuating temperature, $\mathrm{pH}$, cyclic loading and the hydrolytic action of saliva. In vitro material testing should not depend only on static stress load. Thermo-mechanical cyclic loading (TMCL) should be included to add fatigue to the formula to be able to derive clinically relative conclusions ${ }^{(6)}$.

There are two types of computerized manufacturing techniques; subtractive and additive technologies. The subtractive manufacturing (SM), CAD/ CAM (Computer-Aided Design/Computer-Aided Milling), is based on milling blocks of material to reach the desired shape. This technique has high maintenance requirements, manufacturing limitations in complex or convoluted forms and involves a waste byproduct during manufacturing. Aside from the time needed to cut away through the material to reach the desired shape, milling machines usually work in a piece by piece order which substantially increases the time necessary for manufacturing ${ }^{(7)}$.

Additive manufacturing (AM), 3D printing, is considered cutting-edge technology in computerized manufacturing. The concept is dependent on the incremental layering of material and has minimal to no waste byproduct ${ }^{(8)}$. It uses different techniques to fuse materials together to make an object from a 3D model data with the ability to produce interlaced or convoluted forms. Most of these methods are capable of working on multiple pieces simultaneously, which makes them time efficient ${ }^{(7,9)}$. The most common AM techniques used in dentistry are stereolithography (SLA), digital light processing (DLP), liquid crystal display (LCD), material jetting (MJ), selective laser sintering (SLS) ${ }^{(10-12)}$. The CAD data files are exported in a universal exchange format Standard Tessellation Language (STL) ${ }^{(13)}$ The same file format could be used for subtractive and additive manufacturing.
Provisional prosthesis manufactured by $\mathrm{CAD} /$ CAM surpasses the structural properties of those made from conventional resins and attain high mechanical stability due to lack of porosity, voids, and polymerization shrinkage that usually accompanies traditional mixing, packing, and setting ${ }^{(14)}$. CAD/ CAM provisional restorations offer an improvement in color stability and optical properties and decrease the residual monomer release. ${ }^{(15)}$

A great advantage of the digital technique is that the dataset can be reused or easily adjusted in case of fracture or during adaptive treatment modifications like in occlusal reconstructions with an adaptive increase in the VOD (vertical occlusal dimension), maxillofacial surgeries, and temporomandibular joint dysfunction therapies. Implant-Supported Provisional Fixed Dental Prosthesis (ISP-FDP) is another area where this technology is best implemented. CAD/CAM provisional restoration is suggested for long-term interim prosthesis and complex cases ${ }^{(14)}$.

A novel approach to the indirect provisional prosthesis is customized prefabricated trans-mucosal (ISP-FDP). Where provisional prosthesis is prepared ahead of surgery based on a digital workflow ${ }^{(16)}$. The same restorative implant components used for the definitive prosthesis could be initially used for provisionalization, which compensates for guided surgery's additional costs. CAD/CAM or 3D printing are used to fabricate these provisional prostheses ${ }^{(17)}$.

The aim of this study is to evaluate the effect of TMCL on the fracture resistance of ISP-FDP fabricated by CAD/CAM and 3D printing. The null hypothesis of this study was that neither TMCL nor fabrication technique will affect the fracture resistance ISP-FDP.

\section{MATERIAL AND METHODS}

Thirty ISP-FDPs were constructed in this study. Specimens were divided into two main groups, fifteen specimens each, according to fabrication technique. Then each main group was divided into three equal sub-groups according to TMCL duration. 
A sample size of 15 in each main group (5 in each sub-group: zero, 3 and 6 months) has $95 \%$ power to detect a difference between means of $2.14^{(18)}$ with a significance level ( $\alpha$ error) 0.05 for data (t-test or Mann Whitney U test) at 95\% confidence intervals. In $95 \%$ (the power) of those experiments, the P-value will be less than 0.05 , so the results will be deemed "statistically significant". In the remaining $5 \%$ of the experiments, the difference between the means will be deemed not statistically significant ${ }^{(18)}$.

\section{Digital model construction:}

The model was designed digitally in the following procedures. A tetragonal shape with $\mathrm{X}, \mathrm{Y}, \mathrm{Z}$ dimensions $50 \mathrm{~mm} \times 9 \mathrm{~mm} \times 20 \mathrm{~mm}$ was created using meshmixer (San Rafael, CA) to act as a virtual block (initial frame of the model) that will stabilize the fixtures. An STL file was exported of the 3D design to be used in planning the implants and the analog model design for the study set-up.

The virtual block was then imported into implant planning software R2GATE (Megagen -Daegu, South KOREA) to plan implants within its boundaries. Two implants ( $4.0 \times 10 \mathrm{~mm}$ AnyRidge) with their abutments EZ Post 5025 (Megagen - Daegu, South KOREA) were planned parallel to each other. The leveling tool in the software was used to level both implant platforms at the same horizontal level. A distance of $19 \mathrm{~mm}$ from the center of each of their platforms (implant's long axis) was kept. This distance corresponds to the average distance between the center of the mandibular second premolar and second molar ${ }^{(19,20)}$. (Figure 1) The block was exported from R2GATE software with two virtual scan bodies corresponding to the restorative implant positions. The scan bodies will be used for analog model creation. Two virtual abutments, EZ Post 5025 representing hexed $\varnothing 5.0$ stock abutments with $2 \mathrm{~mm}$ collar height and a post length of $5.5 \mathrm{~mm}$, were exported in the exact coordinates of the planned implants to be used for the prosthetic design.

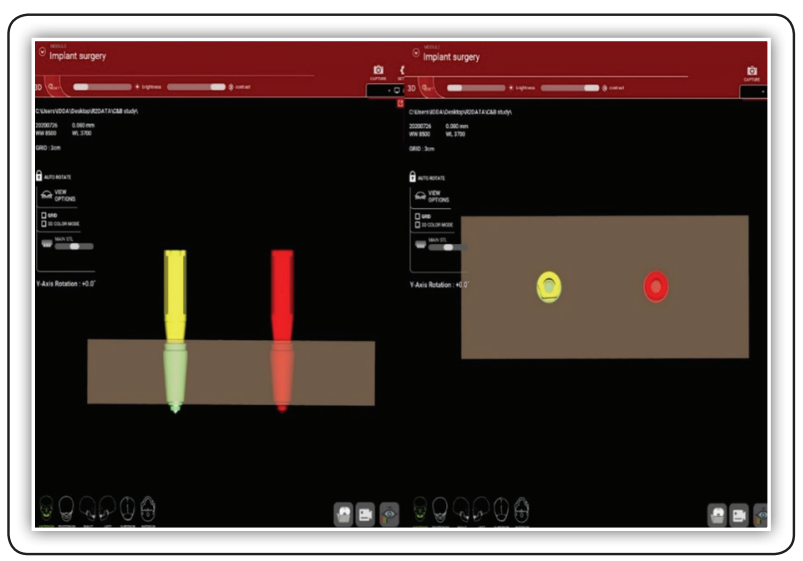

FIG (1) R2Gate: Virtual scan Bodies added to the implants. Two scan bodies were added to represent the implants'3D positions for the future analog model.

The block with the virtual scan bodies exported from R2Gate was imported into the model creator module in Exocad (GmbH, Darmstadt, Germany) along with the digital scan bodies, representing the planned implant positions. The scan bodies were matched to their corresponding parts from the Exocad library. The scan bodies were used to align the digital analogs in the predetermined implant positions. Analog slots were created in the model. Dentifx-3D Modeling HR (Lumi Industries, Montebelluna, Italy) was used to print the models. The printing was performed using the Phrozen Shuffle 3D Printer. The models were printed in $0^{\circ}$ orientation with models' bases towards the printer platform. After the printing was finished, they were cleansed for 3 minutes in a 95\% Isopropyl Alcohol ultrasonic bath to remove any resin residues. According to manufacturer instructions, the models were then left to bench dry and put in the cure box UV Lamp (Troutline, UK) for 30 minutes, according to manufacturer instructions, to make sure they were fully cured. The metal digital-analogs $\varnothing 4.0$ (CANIAR4009) (Megagen - Daegu, South KOREA) were screwed to EZ Post5025 abutments, and the assembly was inserted in the models; the fit was snug. (Figure 3:A)

The virtual block with the digital replica abutments EZ Post 5025 exported from R2GATE (Implant planning software) was used in designing 
the temporary prosthesis. (Figure 2) A cementretained three-unit bridge was designed with the default parameters in Exocad; cement gap $80 \mu \mathrm{m}$, minimal thickness $0.7 \mathrm{~mm}$, the connector was 4.5 $\mathrm{x} 4.3 \mathrm{~mm}$, which is considered higher than the minimum recommendations for most material manufacturers ${ }^{(20-22)}$. The design was exported as an STL file.

The same STL file was used for the fabrication of all the samples. Fifteen samples were printed using the Phrozen Shuffle 3D printer. Slice thickness was $100 \mu \mathrm{m}$, exposure time 6 seconds, bottom exposure time 50 seconds, lifting distance $5 \mathrm{~mm}$ with lifting speed of $65 \mathrm{~mm} / \mathrm{min}$. The printing process lasted about $40 \mathrm{~min}$. The printed IS-FDP were rinsed in isopropyl alcohol $95 \%$ for $3 \mathrm{~min}$ in two rounds. All the samples were then post-cured for $120 \mathrm{~min}^{(23)}$ under UV Lamp (Troutline, UK) according to the manufacturer's recommendations.

The other fifteen samples were milled by the fiveaxis MCX5 CAD/CAM system (MCX5 DENTSPLY Sirona, Charlotte, NC, USA). The milling process lasted about 9 hours. All thirty samples were painted with Optiglaze (196D.644.070, Komet Dental, Lemgo, Germany) and cured for 90 seconds ${ }^{(24)}$.

All the samples were cleaned in an ultrasonic bath with 70\% Isopropyl Alcohol for 3 minutes to ensure proper cleaning of the fitting surface for the cementation. Non-Eugenol Temporary Cement (NETC Meta Biomed CO.LTD, Chungcheonghbukdo, Korea) was used. Excess cement was wiped off with a piece of gauze, and the bridge was placed under a $50 \mathrm{~N}$ of load for 10 minutes $^{(25)}$. (Figure 3: B)

\section{TMCL application:}

Ten samples of each group were subjected to thermo-mechanical cyclic loading via cyclic load multimodal ROBOTA chewing simulator integrated with thermo-cyclic protocol operated on servomotor under a weight of $5 \mathrm{~kg}$, which is comparable to $49 \mathrm{~N}$. In the first five samples (sub-groups B), the test was repeated 37500 times accompanied with 300 thermol cycles $\left(5^{\circ}-55^{\circ} \mathrm{C}\right)$ dwell time 25 seconds to simulate 3 months of aging ${ }^{(6)}$. Another five samples of each group (sub-groups $\mathrm{C}$ ), the test was repeated 75000 times accompanied with 600 thermalcycles $\left(5^{\circ}-55^{\circ} \mathrm{C}\right)$ dwell time 25 seconds to simulate 6 months of aging ${ }^{(6)}$.

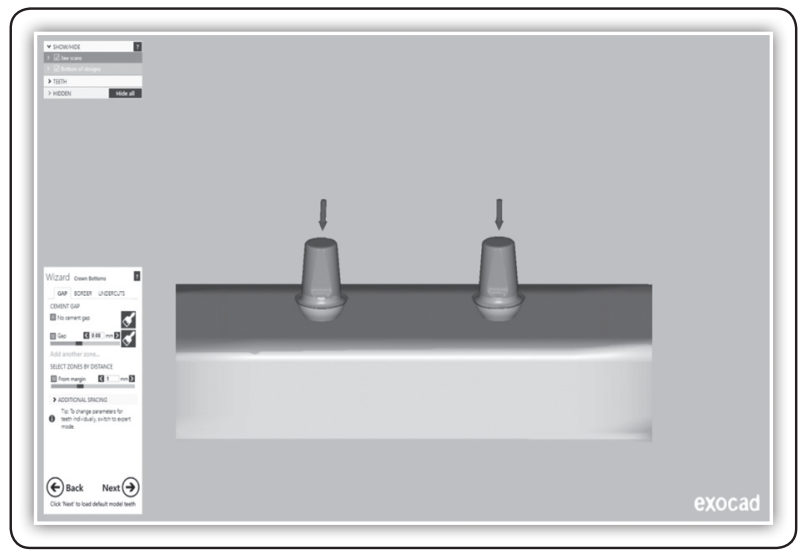

FIG (2) Virtual EZPost 5025 abutments exported in the relative coordinates are used for prosthetic design.

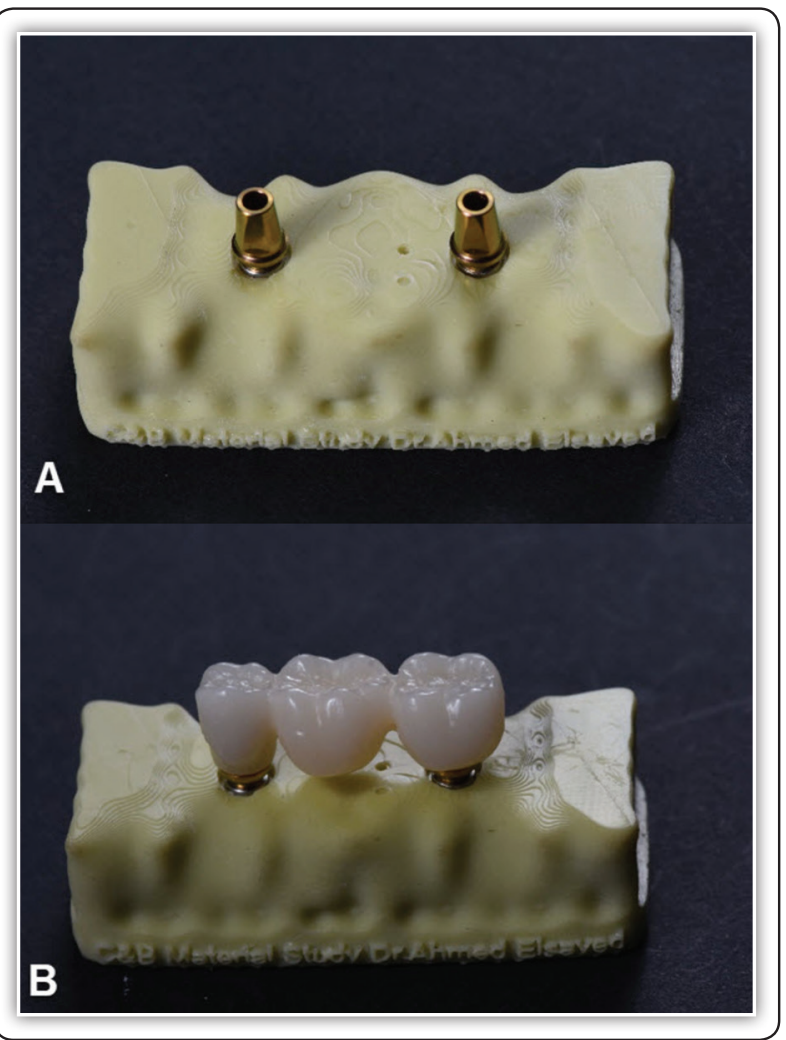

FIG (3) (A) Digital Analog model after analog-abutment attachment. (B) After Bridge Cementation. 


\section{Fracture resistance evaluation:}

This test was performed using a universal fracture testing machine (Bluehill Lite Software from Instron $®)$ all samples were individually mounted on a computer-controlled material testing machine (Model 3345; Instron Industrial Products, Norwood, MA, USA) with a load cell of $5 \mathrm{kN}$ and data were recorded using computer software (Instron ${ }^{\circledR}$ Bluehill Lite Software). Fracture test was done by compressive mode of load applied occlusally using a metallic rod with spherical tip $(5.8 \mathrm{~mm}$ diameter) at a cross-head speed of $1 \mathrm{~mm} /$ min. The load at failure manifested by an audible crack and was confirmed by a sharp drop at loaddeflection curve recorded using computer software (Bluehill Lite Software Instron ${ }^{\circledR}$ Instruments). The load required to fracture was recorded in Newton.

\section{Results \& Statistical Tests:}

Numerical data were explored for normality by checking the data distribution and using normality tests (Kolmogorov-Smirnov and Shapiro-Wilk tests). All data showed a normal (parametric) distribution. Data were presented as mean and standard deviation (SD) values. Two-way Analysis of Variance (ANOVA) was used to study the effect of fabrication technique, thermo-mechanical cycling and their interaction on mean fracture resistance. Bonferroni's post-hoc test was used for pair-wise comparisons when ANOVA test is significant. The significance level was set at $\mathrm{P} \leq 0.05$. Statistical analysis was performed with IBM SPSS Statistics (Version 23.0. Armonk, NY:IBM Corp).

The results showed that the fabrication technique (regardless of thermo-mechanical cycling) had a statistically significant effect on mean fracture resistance (P-value <0.001, Effect size $=0.479)$. Thermo-mechanical cycling (regardless of fabrication technique) also had a statistically significant effect on mean fracture resistance ( $\mathrm{P}$-value $<0.001$, Effect size $=0.743)$. The interaction between the two variables had a statistically significant effect on mean fracture resistance $(\mathrm{P}$-value $=0.014$, Effect size $=0.299)$. Since the interaction between the variables is statistically significant, the variables are dependent upon each other. (Table 1)

Regardless of TMCL, 3D Printing showed statistically significantly higher mean fracture resistance than CAD/CAM (P-value $<0.001$, Effect size $=0.479) .($ Table 2$)$

Regardless of the fabrication technique, there was a statistically significant difference between mean fracture resistance without and with TMCL $($ P-value $<0.001$, Effect size $=0.743)$. (Table 3$)$

Pair-wise comparisons revealed that the mean fracture resistance without TMCL showed the statistically significant highest value. TMCL after three months showed a statistically significant lower mean value. The statistically significant lowest mean fracture resistance was found with TMCL for six months.

TABLE (1) Two-way ANOVA results for the effect of different variables on mean fracture resistance.

\begin{tabular}{|c|c|c|c|c|c|c|}
\hline Source of variation & $\begin{array}{c}\text { Type III Sum } \\
\text { of Squares }\end{array}$ & df & Mean Square & F-value & P-value & $\begin{array}{c}\text { Effect size } \\
\text { (Partial eta } \\
\text { squared) }\end{array}$ \\
\hline Fabrication technique & 81371.875 & 1 & 81371.875 & 22.045 & $<0.001^{*}$ & 0.479 \\
\hline TMCL & 256592.164 & 2 & 128296.082 & 34.757 & $<0.001^{*}$ & 0.743 \\
\hline $\begin{array}{c}\text { Fabrication technique x } \\
\text { TMCL interaction }\end{array}$ & 37787.185 & 2 & 18893.593 & 5.119 & $0.014^{*}$ & 0.299 \\
\hline
\end{tabular}

*: Significant at $P \leq 0.05, D f:$ degrees of freedom $=(n-1)$ 
TABLE (2) The mean, standard deviation (SD) values, and results of two-way ANOVA test for comparison between fracture resistance $(\mathrm{N})$ of the two fabrication techniques regardless of thermo-mechanical cycling.

\begin{tabular}{|c|c|c|c|c|c|}
\hline \multicolumn{2}{|c|}{ 3D Printing } & \multicolumn{2}{c|}{ CAD/CAM } & \multirow{2}{*}{$\begin{array}{c}\text { Effect size } \\
\text { (Partial eta } \\
\text { squared) }\end{array}$} \\
\hline Mean & SD & Mean & SD & & $<0.001 *$ \\
\hline 799.7 & 137 & 695.6 & 92.7 & $<79$ \\
\hline
\end{tabular}

*: Significant at $P \leq 0.05$

TABLE (3) The mean, standard deviation (SD) values, and two-way ANOVA test results for comparison between fracture resistance $(\mathrm{N})$ without and with thermo-mechanical cycling regardless of the fabrication technique.

\begin{tabular}{|c|c|c|c|c|c|c|c|}
\hline \multicolumn{2}{|c|}{ Without TMCL } & \multicolumn{2}{c|}{ TMCL (3 months) } & \multicolumn{2}{c|}{ TMCL (6 months) } & P-value & \multirow{2}{*}{$\begin{array}{c}\text { Effect size } \\
\text { (Partial eta squared) }\end{array}$} \\
\cline { 1 - 5 } Mean & SD & Mean & SD & Mean & SD & & \multirow{2}{*}{8.743} \\
\hline $857.1^{\mathrm{A}}$ & 95.8 & $754.9^{\text {B }}$ & 101.3 & $630.9^{\text {C }}$ & 60.3 & $<0.001^{*}$ & 0.73 \\
\hline
\end{tabular}

*: Significant at $P \leq 0.05$, Different superscripts are statistically significantly different.

Comparison between fracture resistance without and with thermo-mechanical cycling:

With 3D Printing; there was a statistically significant difference between mean fracture resistance without and with thermo-mechanical cycling $(\mathrm{P}$-value $<0.001$, Effect size $=0.722)$. Pair-wise comparisons revealed that the mean fracture resistance without thermo-mechanical cycling showed the highest statistically significant value. Thermomechanical cycling after three months showed a statistically significant lower mean value. The lowest statistically significant mean fracture resistance was found with thermo-mechanical cycling after six months.

With CAD/CAM, there was a statistically significant difference between mean fracture resistance without and with thermo-mechanical cycling (Pvalue $<0.001$, Effect size $=0.420$ ). Pair-wise comparisons revealed that the mean fracture resistance without thermo-mechanical cycling showed the highest statistically significant value. There was no statistically significant difference between thermomechanical cycling after three and six months; both showed the lowest statistically significant mean fracture resistance values, Table 4 and Figure 4.

TABLE (4) The mean, standard deviation (SD) values, and results of two-way ANOVA test for comparison between fracture resistance $(\mathrm{N})$ without and with TMCL with each fabrication technique.

\begin{tabular}{|c|c|c|c|c|c|c|c|c|}
\hline \multirow{2}{*}{$\begin{array}{c}\text { Fabrication } \\
\text { method }\end{array}$} & \multicolumn{2}{|c|}{ Without TMCL } & \multicolumn{2}{|c|}{ TMCL (3 months) } & \multicolumn{2}{c|}{ TMCL (6 months) } & \multirow{2}{*}{ P-value } & \multirow{2}{*}{$\begin{array}{c}\text { Effect size } \\
\text { (Partial eta squared) }\end{array}$} \\
\cline { 2 - 7 } & Mean & SD & Mean & SD & Mean & SD & & 0.722 \\
\hline 3D Printing & $929.9^{\mathrm{A}}$ & 69.8 & $836.3^{\mathrm{B}}$ & 45.1 & $633.1^{\mathrm{C}}$ & 34.6 & $<0.001^{*}$ & 0.420 \\
\hline CAD/CAM & $784.3^{\mathrm{A}}$ & 50.4 & $673.6^{\mathrm{B}}$ & 67.4 & $628.8^{\mathrm{B}}$ & 83.5 & $<0.001^{*}$ & 0 \\
\hline
\end{tabular}

*: Significant at $P \leq 0.05$, Different superscripts are statistically significantly different 


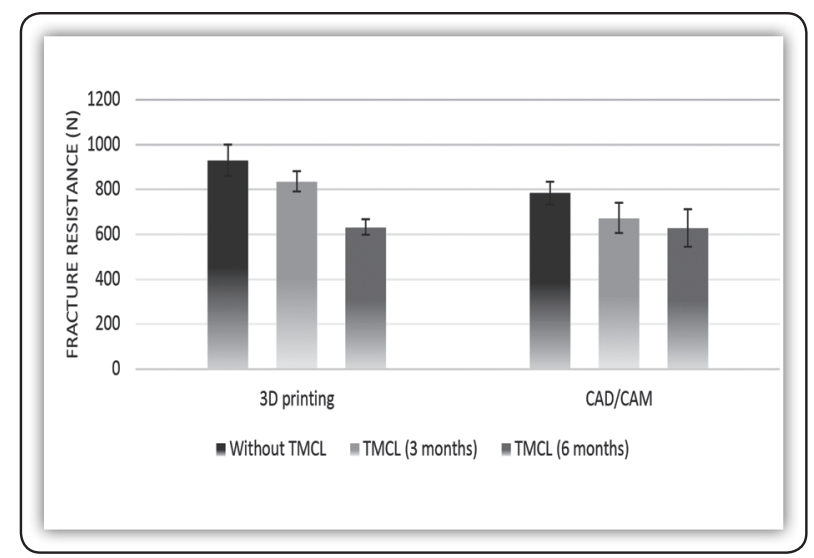

FIG (4) Bar chart representing mean and standard deviation values for fracture resistance of the without and with thermo-mechanical cyclic loading.

\section{DISCUSSION}

The null hypothesis of this study was rejected as fabrication technique and TMCL had an effect on the fracture resistance. Regardless of the TMCL; 3D printed group $(799.7 \mathrm{~N} \pm 37)$ was statistically significantly higher than the milled group (695.6 $\mathrm{N} \pm 92$ ). Pair-wise comparisons revealed that TMCL had a statistically significant effect on both fabrication techniques. Regardless of fabrication technique; the samples without TMCL showed the highest significant value $(857.1 \mathrm{~N} \pm 95.8)$. Where three months TMCL showed a statistically significant lower mean value $(754.9 \pm 101.3)$. The statistically significant lowest mean fracture resistance was found in six months TMCL $(630.9 \pm 60.3)$.

These results are in confinement with the study of Park et al. ${ }^{(26)}$ Other studies showed no significant difference between fabrication techniques ${ }^{(25,27)}$. One study revealed that CAD/CAM had a significant flexural strength compared to $3 \mathrm{D}$ printed resin. (18) This result was attributed to controlled factory fabrication conditions resulting in a material with higher density and lower flow distribution than that produced with $3 \mathrm{D}$ printing.

In the present study, the 3D printed provisional material surpassed the milled group. These conflicting results may be partly due to differences in experimental methods, material types, fabrication processes, and the aging protocol. Tahayeri et al., (27) used the regular NextDent $C \& B$ and skipped the post-printing light polymerization on purpose to examine the core strength of the material immediately after printing ${ }^{(27)}$.

While Reeponahma et al.'s, ${ }^{(25)}$ study was conducted on single crown provisional prosthesis. The current study set-up is based on a three-unit FDP, where the pontic is the load application point, which exposes the material to some kind of complex flexural forces. Finite Element Analysis (FEA) of dental bridge frameworks demonstrated the highest compressive stresses at the occlusal embrasure and highest tensile stresses at the gingival embrasure ${ }^{(28)}$. In the present study the NextDent C\&B MFH (micro-filled hybrid) was used. The different composition of the tested materials explains the variation in results. The filler content was responsible for preventing crack propagation and increasing surface hardness in studies of different materials ${ }^{(9,29)}$.

The approach described in the present study is an evolution of the conventional protocol in a complete digital workflow. ${ }^{(16)}$ A conventional workflow involves a complicated sequence of steps, where potential errors could be incorporated in any of the workflow steps. A minor error early in the process might lead to a more prominent error at the end. Therefore, the use of a digital workflow is easier and faster. Clinical prosthetic digital workflow has been reported to be more efficient for the dentist and more comfortable for the patient than conventional protocols $^{(31)}$.

In this study, a mandibular first molar was used as a pontic since it is the widest mesio-distal tooth in the mouth, which better tests the material's fracture resistance ${ }^{(32,33)}$.

Cement-retained crown design was adopted in this study only for convenience. A cement-retained crown has a more integral occlusal surface, while a screw-retained crown has a screw hole in the 
occlusal surface. Sailer et al., ${ }^{(34)}$ reported a higher incidence of fracture in screw-retained single crowns than cement-retained and preferred the usage of cement-retained ${ }^{(34)}$. The findings were also in confinement with the results of Nissan et al., ${ }^{(35)}$ who indicated that the long-term outcome of cement-retained was superior to that of screwretained clinically and biologically ${ }^{(35)}$. In contrast, other studies determined no significant difference between cement or screw-retained crowns in both technical and clinical failures ${ }^{(36,37)}$. Using a cementretained bridge relatively does not affect this study since the point of force application is the pontic.

Thermo-mechanical cyclic loading (TMCL) can simulate the dynamic nature of the oral conditions and the occlusal forces during the provisionalization period in a laboratory setup ${ }^{(6,18)}$. TMCL was performed on elected sub-groups (B \& C) of both fabrication techniques to simulate oral conditions during common temporization periods. A single subgroup (A) of each technique was left without TMCL to represent a baseline of the material strength. None of the aged groups presented an early failure during thermo-mechanical cyclic loading.

After TMCL for three months, 3D printed group maintained a higher mean fracture resistance than the milled group. However, there was no statistically significant difference between the mean fracture resistances of the two techniques after six months of TMCL. The aging protocol has demonstrated that three months of TMCL is responsible for a $10 \%$ reduction in fracture resistance in $3 \mathrm{D}$ printed samples and a $14 \%$ reduction in milled samples. Six months of TMCL lead to a $31.9 \%$ reduction in 3D printed specimens and a $19.8 \%$ reduction in milled samples. It was also observed that $3 \mathrm{D}$ printed groups showed more brittle fracture than the milled groups. TMCL induced more brittleness in both groups.

Cyclic mechanical and thermal loads induce cumulative degradation, crack initiation, and growth, resulting in catastrophic failure of dental prostheses. Voids introduced during material processing, imperfect interfaces, and residual stresses might propagate this failure ${ }^{(1,8,15)}$. Fractures occur when the stress concentration inside the material reaches the critical level known as the "plane strain fracture toughness" $\mathrm{K}_{\mathrm{I}} \mathrm{C}$. Characterization $\mathrm{K}_{\mathrm{I}} \mathrm{C}$ can thus help prevent catastrophic failures ${ }^{(29)}$.

Introduction of fillers that arrest crack lines is one of the methods used in increasing the fracture resistance of dental resin. The filler particles act as blockades in the way of crack lines leading to their pinning and bowing between these particles ${ }^{(30)}$. Consequently, increasing the fracture energy and plane strain fracture toughness for this material. In addition, a high degree of conversion and isotropy positively influence the fracture resistance of the material ${ }^{(29)}$.

Conversely, wet storage or accelerated aging have hydrolytic actions, which induce the pull out of these filler particles by breaking down the silane bond between the fillers and the resin. It also has a plasticizing effect on the resin matrix ${ }^{(29,38)}$.

Thermo-mechanical cycling induced further embrittlement of the material which might be due to heat-induced maturation of the polymerization reaction in the material. Incomplete polymerization occurs as a result of entrapment of some active components in the polymer network such as free radicals, active monomers and photoinitiators during the polymer vitrification stage. Some studies have noticed the continuation of polymerization when composite resins are heated near their glass transition $[\mathrm{Tg}]$ temperature. Also, under the presence of a solvent like water, the mobility of the system increases, leading to the diffusion of these entrapped particles and causing radical recombination to occur ${ }^{(39)}$.

Chung et al ${ }^{(40)}$ observed superficial changes and matrix degradation using SEM (scanning electron microscope) with thermocycling. They concluded that composite resins are indigenously brittle and their brittleness increases with aging ${ }^{(40)}$. 
Both fabrication techniques are viable for fabricating provisional prostheses because the fracture load of all the samples included in this study exceeds the registered normal physiological chewing force in humans $110-125 \mathrm{~N}^{(41,42)}$. The samples also showed the ability to withstand the maximum bite force in anterior and posterior teeth 230-698 $\mathrm{N}^{(43)}$. However, long-standing provisional prostheses might experience a load failure in parafunctional cases, especially ones that serve up to 6 months of provisionalization.

This study was completed in-vitro, which is considered one of its limitations. In-vivo studies present better clinical relevance for the presence of more chemical and physical challenges such saliva (enzymes and $\mathrm{pH}$ ), acidic drinks, hard food and tooth brushing. Another limitation of this study is the range of material in each group, which is limited to a single manufacturer due to financial limitations. Incorporating several manufacturers is suggested in future studies to evaluate the effect of the manufacturing role and drive an absolute conclusion that fabrication technique has a significant influence on fracture strength.

\section{CONCLUSIONS}

Based on the findings of this in vitro study, the following conclusions were drawn:

1. Fabrication technique and thermos-mechanical cyclic loading have a significant effect on the fracture resistance of implant-supported provisional FDP.

2. 3D Printed provisional prosthesis is a competitive alternative to $\mathrm{CAD} / \mathrm{CAM}$ prosthesis.

3. Both fabrication techniques could be used to fabricate temporary restoration for implant cases up to six months in patients with normal physiologic occlusion.

4. Longer Cycles of Thermo-mechanical cyclic loading have a more prominent effect on the fracture resistance of provisional prosthesis and the amount of degradation is not constant.

\section{REFERENCES}

1. Alt V, Hannig M, Wöstmann B, Balkenhol M. Fracture strength of temporary fixed partial dentures: $\mathrm{CAD} /$ CAM versus directly fabricated restorations. Dent Mater. 2011;27(4):339-47.

2. Heying JJ. Flexural strength of interim fixed prosthesis materials after simulated function. Univ IOWA. 2009;94.

3. Burns DR, Beck DA, Nelson SK. A review of selected dental literature on contemporary provisional fixed prosthodontic treatment: Report of the Committee on Research in Fixed Prosthodontics of the Academy of Fixed Prosthodontics. J Prosthet Dent. 2003;90(5):474-97.

4. Rayyan MM, Aboushelib M, Sayed NM, Ibrahim A, Jimbo $\mathrm{R}$. Comparison of interim restorations fabricated by $\mathrm{CAD} /$ CAM with those fabricated manually. J Prosthet Dent. 2015;114(3):414-9.

5. Patzelt SBM, Bahat O, Reynolds MA, Strub JR. The allon-four treatment concept: a systematic review. Clin Implant Dent Relat Res. 2014 Dec;16(6):836-55.

6. Nawafleh N, Hatamleh M, Elshiyab S, Mack F. Lithium Disilicate Restorations Fatigue Testing Parameters: A Systematic Review. J Prosthodont. 2016;25(2):116-26.

7. Van Noort R. The future of dental devices is digital. Dent Mater. 2012;28(1):3-12.

8. Ligon SC, Liska R, Stampfl J, Gurr M, Mülhaupt R. Polymers for 3D Printing and Customized Additive Manufacturing. Chem Rev. 2017;117(15):10212-90.

9. Kessler A, Reymus M, Hickel R, Kunzelmann KH. Threebody wear of 3D printed temporary materials. Dent Mater. 2019;35(12):1805-12.

10. Alharbi N, Osman R, Wismeijer D. Effects of build direction on the mechanical properties of 3D-printed complete coverage interim dental restorations. J Prosthet Dent. 2016;115(6):760-7.

11. Moon W, Kim S, Lim BS, Park YS, Kim RJY, Chung SH. Dimensional accuracy evaluation of temporary dental restorations with different $3 \mathrm{~d}$ printing systems. Materials (Basel). 2021;14(6):1-9.

12. Chen H, Cheng DH, Huang SC, Lin YM. Comparison of flexural properties and cytotoxicity of interim materials printed from mono-LCD and DLP 3D printers. J Prosthet Dent. 2020;1-6.

13. Revilla-León M, Sadeghpour M, Özcan M. An update on applications of 3D printing technologies used for processing polymers used in implant dentistry. Odontology. 2020;108(3):331-8. 
14. Edelhoff D, Beuer F, Schweiger J, Brix O, Stimmelmayr M, Guth J-F. CAD/CAM-generated high-density polymer restorations for the pretreatment of complex cases: a case report. Quintessence Int. 2012;43(6):457-67.

15. Alp G, Murat S, Yilmaz B. Comparison of Flexural Strength of Different CAD/CAM PMMA-Based Polymers. J Prosthodont. 2019;28(2):e491-5.

16. Stapleton BM, Lin WS, Ntounis A, Harris BT, Morton D. Application of digital diagnostic impression, virtual planning, and computer-guided implant surgery for a CAD/ CAM-fabricated, implant-supported fixed dental prosthesis: A clinical report. J Prosthet Dent. 2014;112(3):402-8.

17. Joda T, Ferrari M, Gallucci GO, Wittneben JG, Brägger U. Digital technology in fixed implant prosthodontics. Periodontol 2000. 2017;73(1):178-92.

18. Digholkar S, Madhav VNV, Palaskar J. Evaluation of the flexural strength and microhardness of provisional crown and bridge materials fabricated by different methods. J Indian Prosthodont Soc. 2016;16(4):328-34.

19. Bonfante EA, Coelho PG, Navarro JM, Pegoraro LF, Bonfante G, Thompson VP, et al. Reliability and failure modes of implant-supported Y-TZP and MCR three-unit bridges. Clin Implant Dent Relat Res. 2010;12(3):235-43.

20. Vafaee F, Firouz F, Khoshhal M, Hooshyarfard A, Shahbazi A, Roshanaei G. Fatigue Fracture Strength of ImplantSupported Full Contour Zirconia and Metal Ceramic Fixed Partial Dentures. J Dent (Tehran). 2017;14(3):165-72.

21. Beuer F, Schweiger J, Edelhoff D. Digital dentistry: an overview of recent developments for CAD/CAM generated restorations. Br Dent J. 2008 May;204(9):505-11.

22. Alt V, Hannig M, Wöstmann B, Balkenhol M. Fracture strength of temporary fixed partial dentures: CAD/ CAM versus directly fabricated restorations. Dent Mater. 2011;27(4):339-47.

23. Park S, Park J, Kim S, Heo S, Koak J. Comparison of Flexural Strength of Three-Dimensional Printed Three-Unit Provisional Fixed Dental Prostheses according to Build Directions. 2019;12(1):13-9.

24. Şahin O, Dede DÖ, Körołlu A, Yilmaz B. Influence of surface sealant agents on the surface roughness and color stability of artificial teeth. J Prosthet Dent. 2015;114(1):130-7.

25. Reeponmaha T, Angwaravong O, Angwarawong T. Comparison of fracture strength after thermomechanical aging between provisional crowns made with CAD/CAM and conventional method. J Adv Prosthodont. 2020;12(4): 218-24.
26. Park SM, Park JM, Kim SK, Heo SJ, Koak JY. Flexural strength of 3D-printing resin materials for provisional fixed dental prostheses. Materials (Basel). 2020;13(18): $1-14$.

27. Tahayeri A, Morgan M, Fugolin AP, Bompolaki D, Athirasala $\mathrm{A}$, Pfeifer $\mathrm{CS}$, et al. 3D printed versus conventionally cured provisional crown and bridge dental materials. Dent Mater. 2018 Feb;34(2):192-200.

28. Oh W, Götzen N, Anusavice KJ. Influence of connector design on fracture probability of ceramic fixed-partial dentures. J Dent Res. 2002;81(9):623-7.

29. Drummond JL. Degradation, fatigue, and failure of resin dental composite materials. J Dent Res. 2008 Aug $1 ; 87(8): 710-9$

30. Kerby RE, Knobloch LA, Sharples S, Peregrina A. Mechanical properties of urethane and bis-acryl interim resin materials. J Prosthet Dent. 2013 Jul;110(1):21-8.

31. Mangano F, Veronesi G. Digital versus analog procedures for the prosthetic restoration of single implants: A randomized controlled trial with 1 year of follow-up. Biomed Res Int. 2018;2018(August).

32. Park DE, Kim HK, Lim YS, Nakatsuka M, Kwon HB, Han $\mathrm{SH}$, et al. Different mandibular first molar shapes according to groove and cusp configuration in relation to suggested bracket position. Eur J Orthod. 2013;35(6):730-6.

33. Malkoç S, Basçiftçi FA, Nur M, Catalbas B. Maxillary and mandibular mesiodistal tooth sizes among different malocclusions in a sample of the Turkish population. Eur $\mathbf{J}$ Orthod. 2011 Oct;33(5):592-6.

34. Sailer I, Mühlemann S, Zwahlen M, Hämmerle CHF, Schneider D. Cemented and screw-retained implant reconstructions: a systematic review of the survival and complication rates. Clin Oral Implants Res. 2012 Oct;23:163201.

35. Nissan J, Narobai D, Gross O, Ghelfan O, Chaushu G. Long-term outcome of cemented versus screw-retained implant-supported partial restorations. Int J Oral Maxillofac Implants. 2011;26(5):1102-7.

36. Yazigi C, Kern M, Chaar MS, Libecki W, Elsayed A. The influence of the restorative material on the mechanical behavior of screw-retained hybrid-abutment-crowns. J Mech Behav Biomed Mater. 2020;103988.

37. Cacaci C, Cantner F, Mücke T, Randelzhofer P, Hajtó J, Beuer F. Clinical performance of screw-retained and cemented implant-supported zirconia single crowns: 36-month results. Clin Oral Investig. 2017;21(6):1953-9. 
38. Takeshige F, Kawakami Y, Hayashi M, Ebisu S. Fatigue behavior of resin composites in aqueous environments. Dent Mater. 2007 Jul;23(7):893-9.

39. Ghavami-lahiji M, Firouzmanesh M, Bagheri H, Kashi TSJ, Razazpour F, Behroozibakhsh M. resin composite Pr ov isi Pr ov isi Pr ov isi Pr ov isi on. 2018;43(2):3-9.

40. Chung SM, Yap AUJ, Chandra SP, Lim CT. Flexural strength of dental composite restoratives: comparison of biaxial and three-point bending test. J Biomed Mater Res B Appl Biomater. 2004 Nov;71(2):278-83.
41. Fontijn-Tekamp FA, Slagter AP, Van Der Bilt A, Van 'T Hof MA, Witter DJ, Kalk W, et al. Biting and Chewing in Overdentures, Full Dentures, and Natural Dentitions. J Dent Res. 2000 Jul 1;79(7):1519-24.

42. Helkimo E, Carlsson GE, Helkimo M. Bite force and state of dentition. Acta Odontol Scand. 1977 Jan 1;35(6): 297-303.

43. Serra T, Planell JA, Navarro M. High-resolution PLAbased composite scaffolds via 3-D printing technology. Acta Biomater. 2013;9(3):5521-30. 